\title{
Epithelial to mesenchymal transition in hepatocellular carcinoma
}

\author{
W Mikulits
}

\section{Abstract}

The transition of epithelial cells to a mesenchymal phenotype (EMT) is of paramount relevance for embryonic development and adult wound healing. During the past decade, EMT has been increasingly recognized to occur during the progression of various carcinomas such as hepatocellular carcinoma (HCC). Here we focus on EMT in both experimental liver models and human HCC, emphasizing the underlying molecular mechanisms which show partial recurrence of embryonic programs such as TGF- $\beta$ and Wnt/ $\beta$-catenin signaling including collaboration with hepatitis viruses. We further discuss the differentiation repertoire of malignant hepatocytes with respect to the potential acquisition of stemness, and the involvement of the mesenchymal to epithelial transition, the reversal of EMT, in cancer dissemination and metastatic colonization. The strong evidence for EMT in HCC patients demands novel strategies in pathological assessments and therapeutic concepts to efficiently combat HCC progression.

\section{Keywords}

Hepatocyte; HCC; EMT; tumor progression; cell invasion; metastasis; tumor-stroma; stemness

Hepatocellular carcinoma (HCC) accounts for more than five percent of all cancer cases and is the fifth leading cause of cancer mortality worldwide [1,2]. The etiology of HCC includes major risk factors such as viral infection with hepatitis B or C (HBV, HCV), dietary exposure to fungal aflatoxin or alcohol intoxication [3]. Independent of the carcinogenic insult, chronic hepatitis and cirrhosis resulting from inflammation and fibrosis are present in almost eighty percent of HCC cases worldwide [2,4]. Additional etiological factors underlying fibrosis and cirrhosis such as hereditary hemochromatosis or non-alcoholic fatty liver disorders have also a potential impact in the development of HCC $[5,6]$. The cirrhotic lesions that commonly precede dysplastic HCC-like foci and nodules are prone to acquire initial genomic alterations which further accumulate during hepatocarcinogenesis $[7,8]$. About $80 \%$ of HCC patients are diagnosed at an advanced stage of disease due to the lack of symptoms during early stages of $\mathrm{HCC}$ and the rapid progression of disease [9]. In general, liver resection and orthotopic liver transplantation are considered as the only curative treatments of $\mathrm{HCC}$, and the prognosis of $\mathrm{HCC}$ is poor when the tumor burden cannot be surgically removed [10]. Despite major efforts in past decades, therapeutic options to interfere with HCC progression are very limited and novel therapeutic strategies for efficient treatment are needed.

Hepatocytes or even progenitor cells of the liver represent the cellular origin of HCC. In particular, epithelial hepatocytes are mitotically inactive under physiological conditions and highly differentiated with respect to their specialized functions for e.g. glucose, amino acid and lipid metabolism, but show an extraordinary proliferative capacity for regeneration in response to damaging stimuli such as partial hepatectomy or chemical intoxication [11,12]. Interestingly, serial transplantation experiments in mice showed that differentiated hepatocytes can be stimulated to more than 70 cell doublings after liver injury, demonstrating their vast proliferative abilities for tissue repair and repopulation [13]. In the 
pathogenesis of HCC, multiple and diverse mechanisms have been reported to cause the aberrant proliferation and dedifferentiation of hepatocytes which lead to the subsequent development of malignant neoplasia. Most frequently, (i) inactivation of tumor suppressors such as $\mathrm{p} 53$, retinoblastoma or the CDKN2A-encoded proteins $\mathrm{p} 14^{\mathrm{ARF}}$ and $\mathrm{p} 16^{\mathrm{Ink}} 4 \mathrm{~A}$, (ii) overexpression of cyclin D1/Cdk4, insulin-like growth factor-II or c-MET as well as (iii) activation of the Ras/mitogen activated protein kinase (MAPK), transforming growth factor (TGF)- $\beta$ signaling or Wnt/ $\beta$-catenin signaling have been described as critical events in HCC $[1,7,8,14,15]$.

The sequence of genetic and epigenetic alterations required for malignant growth of hepatocytes appears to be random in the development of HCC from dysplastic foci to large tumor nodules. Yet, the escape of carcinoma cells from the solid tumor might be due to dedifferentiation of epithelial cells which occurs by loss of cell-to-cell contacts and the concomitant gain of migratory and invasive abilities [16]. This phenotypical conversion of cells, collectively designated as epithelial to mesenchymal transition (EMT), has been described in different types of carcinoma cells including HCC [17-19]. Accumulating evidence suggests that hepatocellular EMT plays a pivotal role in the dissemination of malignant hepatocytes during HCC progression. In this review, we provide a summary on the epithelial plasticity of hepatocytes during the development of malignant liver disease by outlining well-characterized models of hepatocellular EMT and by focusing on recent observations of EMT in HCC patients. Furthermore, we briefly discuss the relevance of EMT for the pathological assessment of HCC progression and intrahepatic metastasis as well as for novel anti-cancer strategies.

\section{EMT in development and tissue repair}

EMT is a transient and reversible switch from a polarized, epithelial to a fibroblastoid or mesenchymal cellular phenotype, the latter exhibiting highly motile and invasive properties $[19,20]$. Cells that have undergone EMT are endowed with the ability to transmigrate basement membranes and stromal tissues as well as to intravasate and pass the circulatory system. In general, this process is a central event in both early stages of embryonic development and in pathophysiological situations such as wound healing, chronic inflammation and carcinoma progression (Figure 1). During early embryonic development, epithelial cell sheets convert into mesenchymal cells which is the basis for the formation of a three-layered embryo, a process known as gastrulation [19,21]. In mammals, EMT takes place at the blastula stage during parietal endoderm formation, where epithelial precursors of the visceral endoderm get in contact with the mural trophectoderm in the marginal zone and generate newly mesodermal mesenchymal cells which later contribute to the extraembryonic tissues. Moreover, EMT also shows fundamental implications in organogenesis during formation of the heart, the musculoskeletal system, craniofacial structures and the peripheral nervous system.

EMT mechanisms are further active during wound healing, where the response to injury reduces the adhesiveness of epithelial cells in the epidermis through complex cellular and molecular interactions, and increases the migratory phenotype in order to re-generate intact epithelial sheets [22]. EMT is further involved in the fibroproliferative wound healing known as fibrosis, which is associated with chronic inflammation and represents a nonregenerative repair of the organ. Besides fibrosis in kidney and lung, EMT has been demonstrated to significantly contribute to liver fibrosis. In particular, Zeisberg et al. showed that mouse hepatocytes convert to fibroblast-specific protein (FSP)-1-positive hepatic fibroblasts via TGF- $\beta 1$-induced EMT which could be blocked by administration of bone morphogenic protein (BMP)-7. In addition to hepatic stellate cell (HSC)-derived myofibroblasts, portal myofibroblasts and bone-marrow-derived mesenchymal stem cells, 
these data provided first evidence that hepatocyte-derived fibroblasts are a significant source of mesenchymal cells that contribute to progression of liver fibrosis [23,24]. Remarkably, a significant portion of FSP-1/a-smooth muscle actin-positive fibroblasts (about 10\%) have been demonstrated to derive from hepatocytes via EMT, suggesting a substantial population of hepatocytes involved in fibrogenesis. A recent study by Dooley et al. aimed at clarifying the role of hepatocytes in hepatic fibrosis, and demonstrated that primary mouse hepatocytes lose their epithelial phenotype in response to TGF- $\beta$ by loss of E-cadherin, a major component of epithelial adherens junctions, and upregulation of snail (SNAII) which represents a potent transcriptional repressor of E-cadherin [25]. Similar results were obtained in further studies by employing immortalized murine AML-12 hepatocytes which show TGF- $\beta$-dependent EMT that is associated with activation of Smad $2 / 3$ signaling and collagen I synthesis as well as induction of snail [26]. This EMT phenotype and profibrogenic gene expression signature could be attenuated by Smad7 which antagonizes TGF$\beta /$ Smad signaling or RNA interference against Smad4, suggesting that hepatocytes undergo EMT-like phenotypic changes in response to TGF- $\beta$ and actively contribute to fibrogenesis $[25,26]$. In primary murine hepatocytes, the EMT was associated with a focal adhesion kinase (FAK)/Src-dependent activation of phosphoinositide-3 kinase (PI3K)/Akt and Erk1/2 signaling causing resistance to apoptosis and induction of EMT, respectively [27]. These results confirmed recent data obtained by the TGF- $\beta$-mediated EMT of immortalized MMHhepatocytes which require Src/FAK for delocalization of membrane-bound E-cadherin and transcriptional up-regulation of mesenchymal markers [28]. Further studies revealed that hepatocytes isolated from cirrhotic livers, which are exposed to sustained TGF- $\beta$ levels in vivo, differ in their phenotype from hepatocytes of healthy mouse livers by showing elongated, fibroblastoid cells expressing vimentin and collagen I [29]. Thus, chronic inflammation and fibrosis with increased TGF- $\beta$ expression promotes hepatocytes to change their phenotype and functions in order to escape apoptosis which is the basis for hepatocarcinogenesis. In this respect, studies on fetal rat hepatocytes that have undergone EMT upon TGF- $\beta$ treatment have demonstrated that EMT leads to the resistance against TGF- $\beta$-mediated apoptosis. Survival of fibroblastoid derivatives of hepatocytes might be accomplished by the induction of epidermal growth factor (EGF)-R- and Src-dependent activation of PI3K/Akt as well as by high levels of Bcl- $\mathrm{X}_{\mathrm{L}}$ and snail [30,31].

\section{EMT in murine hepatocellular tumor models}

Hepatocyte nuclear factor (HNF)-4, a transcription factor essential for hepatocyte differentiation, has been implicated in the EMT of slow-growing mouse HCC cells to highly invasive, fast-growing and dedifferentiated cells. EMT associated with loss of cell polarity, decrease in cell-cell and cell-ECM adhesion was coupled to reduced HNF-4 expression [32]. Restoration of HNF-4a 1 expression in fast-growing HCC cells reversed the progressive phenotype by re-establishing hepatocytes with intact epithelial organization and reduced proliferation, indicating that loss of HNF-4 is an important determinant of HCC progression.

Immortalized MMH hepatocytes, expressing the cytoplasmic domain of c-Met, or

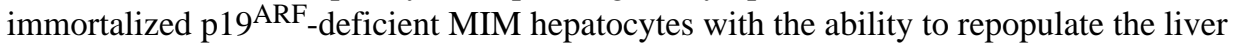
have been further employed to establish murine EMT tumor models [33,34]. Both MMH and MIM hepatocytes have been reported to show EMT caused by the synergy between oncogenic Ras and TGF- $\beta 1$ signaling - but also through the cooperation of Ras with TGF$\beta 2$ or TGF- $\beta 3$ - which leads to a dramatic progression in malignancy towards metastasis (Figure 2) [35,36]. These changes in epithelial plasticity associate with cytoplasmic dislocation or loss of E-cadherin, ZO-1, p120 catenin, and desmoplakin as well as the activation of Smad2/3 signaling leading to autocrine secretion of TGF- $\beta$. Thus, TGF- $\beta$ provides a dual role in hepatocarcinogenesis since it triggers cell cycle arrest and apoptosis in the healthy liver and during tumor initiation, and in contrast, is capable to control 
dedifferentiation and spreading of neoplastic hepatocytes by induction of EMT (Figure 2). Studies of EMT kinetics revealed that the induction phase of EMT involves the crosstalk between TGF- $\beta$ and MAPK, and showed that the maintenance phase depends on the additional activation of PI3K/Akt [37]. In the immediate early phase of EMT induction, cFos expression has been observed to be transiently upregulated [35]. Sustained AP-1 induction through continuous expression of c-Fos resulted in proliferation arrest and cell death of hepatocytes [38], demonstrating that c-Fos mediates tumor-suppressive effects that could be prevented either by scavenging reactive oxygen species or by expressing antiapoptotic $\mathrm{Bcl}-\mathrm{X}_{\mathrm{L}}$. In this line, c-Fos expression exhibited a strong negative interference with tumorigenesis of hepatocytes in vitro and in vivo, indicating that c-Fos acts as a negative regulator of hepatocarcinogenesis downstream of TGF- $\beta$ signaling [38].

Independent of the genetic background of EMT, platelet-derived growth factor (PDGF-)A ligand and both PDGF receptor (PDGF-R) $\alpha$ and $\beta$ were found to be upregulated along with autocrine PDGF secretion [35]. Inhibition of PDGF signaling revealed decreased migration in vitro and efficient tumor suppression in vivo, indicating an essential role of PDGF in TGF- $\beta$-mediated EMT of neoplastic hepatocytes. Upregulation of PDGF/PDGF-R has been further reported through the collaboration of oncogenic Ras and interleukin-like EMT inducer (ILEI) during the fibroblastoid conversion of Ras-transformed hepatocytes [39]. In addition, ILEI - a downstream target of TGF- $\beta$ during hepatocellular EMT - collaborates with oncogenic Ras to induce both the activation signal transducer and activator of transcription (STAT) 3 and the nuclear accumulation of $\beta$-catenin via PDGF/PDGF-R. Importantly, interference with PDGF-R signaling abolishes nuclear $\beta$-catenin translocation upon TGF- $\beta$ signaling in vivo [40]. This finding is of particular relevance since about $33-67 \%$ of HCC cases display nuclear accumulation of $\beta$-catenin [41]. Studies on the tumorstroma crosstalk revealed that the paracrine regulation by myofibroblasts is responsible for the TGF- $\beta$-dependent induction as well as the PDGF-mediated maintenance of EMT (Van Zijl et al., 2009, manuscript submitted). Analysis of Wnt/ $\beta$-catenin further showed that nuclear $\beta$-catenin in neoplastic hepatocytes correlates with their transdifferentiation to early hepatocyte progenitors that abundantly express M2-pyruvate kinase (M2-PK) and cytokeratin (CK)19, but lack epithelial CK8 and CK18. On the contrary, ablation of $\beta$ catenin by the overexpression of axin caused strong expression of CK8 and CK18 (Zulehner et al., 2009, manuscript submitted). These data demonstrate that the TGF- $\beta$-dependent, nuclear accumulation of $\beta$-catenin results in a loss of epithelial markers and increased expression of hepatic progenitor markers.

\section{EMT in HCC patients}

While hepatocytes of well differentiated human HCC samples and adjacent non-cancerous liver parenchyma show E-cadherin at the plasma membranes, cytoplasmic localization or frequent loss of E-cadherin is displayed in poorly differentiated HCC (Figure 3). These data suggest a disruption of E-cadherin/ $\beta$-catenin complexes at cell boundaries that is characteristic for hepatocellular EMT and comparable to observation of experimental HCC in mouse. A recent immunohistochemical study revealed that $58 \%$ of human $\mathrm{HCC}$ cases showed reduced levels of E-cadherin along with a weakened expression of p120 catenin, $a-$ catenin, $\beta$-catenin and $\gamma$-catenin (plakoglobin) in a comparable percentage of HCC patients [42]. The reduced expression of E-cadherin was accompanied by (partial) nuclear translocation of $\beta$-catenin, and significantly correlated with intrahepatic metastasis and poor survival of patients. In the same line, E-cadherin was reported to be strongly expressed at cell-to-cell contacts in the non-metastatic HCC patient group, whereas only few cells displayed very weak staining of E-cadherin at the cell surface in the metastatic patients group [43]. Expression of Twist, a negative regulator of E-cadherin transcription, has been reported in a large proportion of primary $\mathrm{HCC}$ and metastasis which was negatively 
correlated with E-cadherin expression [44]. Human HCC cell lines exhibiting either a low or high metastatic potential confirmed these data. Expression of Twist in non-metastatic HCC cells induced EMT, leading to strong reduction of E-cadherin and increased invasion. Immunohistochemical analysis of another human HCC selection showed elevated expression of the extracellular matrix (ECM) protein laminin (Ln)-5 with a concomitant downregulation of E-cadherin and dissociation of $\beta$-catenin from cell borders particularly at the tumor invasive front which correlated also with snail and slug (SNAI2) expression [45]. Experiments using HCC cell lines showed that Ln-5 in collaboration with TGF- $\beta$ was able to induce a complete EMT which could be reversed to MET via anti-a 3 integrin blocking antibody. Further studies employing HCC cell lines revealed that Ln-5 causes the phosphorylation of $\beta 4$ in a $6 \beta 4$ integrin which subsequently activates Erk1/2. The phosphorylation of Erk1/2 is required for cell proliferation, however, it depends on the presence of a $3 \beta 1$ integrin [46]. In addition, phosphorylation of threonine residues 788 and 789 of the $\beta 1$ subunit of a $5 \beta 1$ integrin by the TGF- $\beta$ I receptor was found to be necessary for vascular invasion [47].

A switch from E-cadherin expressed at intercellular junctions of normal hepatocytes to mesenchymal T-cadherin during malignant transformation has been detected in a distinct proportion of HCC [48]. The cadherin switch of E- to T-cadherin at the leading edge of cancerous hepatocytes in HCC with vascular infiltration could be connected to enhanced migration and an increased invasive potential, suggesting a role of T-cadherin in metastasis of HCC. Interestingly, the tetraspanin transmembrane 4 superfamily (TM4SF) protein has been demonstrated to be overexpressed in HCC patients. The expression in HCC cell lines caused an increase and cytosolic stabilization of $\mathrm{p} 27^{\mathrm{Kip} 1}$ expression together with an inactivation of RhoA [49]. TM4SF-dependent molecular changes result in aberrant cell cycle progression, loss of E-cadherin and EMT. Microarray analysis of primary HCC versus intrahepatic metastatic HCC revealed upregulation of the transcription factor SOX4 and identified a large number of genes in intrahepatic metastasis with SOX4 consensus promoter binding sites [50]. SOX4 or its target genes including neuropilin 1 and semaphorin 3C enforced migration of HCC cell lines, while RNA interference against SOX4 resulted in a mesenchymal to epithelial (MET)-like phenotype, suggesting that SOX4 is a crucial regulator of HCC invasion and metastasis. Furthermore, microRNA (miR)-122 has been identified as a potent tumor suppressor that is downregulated in intrahepatic metastatic HCC [51]. This is of particular relevance since intrahepatic metastases were observed in about $30 \%$ of cases after surgical removement of small HCC nodules and in $80 \%$ of HCC autopsy cases [52]. Either restoration of miR-122 expression or knockdown of its identified target gene ADAM17 (metalloprotease 17, a sheddase) in metastatic HCC cell lines leads to reduction of mesenchymal markers such as vimentin and decreased migration and invasion in vitro as well as diminished proliferation, angiogenesis and intrahepatic metastasis in vivo.

Recent studies analyzed the cooperative role of viral proteins of HBV and HCV with cellular signaling events during HCC progression. Importantly, the natural HCV core variants isolated from HCV-related HCC tissues have been shown to diminish TGF- $\beta$ / Smad3-dependent growth inhibition and apoptosis, rendering hepatocytes to become less sensitive to tumor-suppressive effects of TGF- $\beta$ [53]. In particular, HCV core proteins facilitate tumor-promoting effects of TGF- $\beta$ by lowering Smad3, allowing primary human hepatocytes and human HCC cells to undergo EMT via strong decrease of E-cadherin. The collaboration of oncogenic signals with viral proteins has been further observed in clinical samples of HCC with chronic HBV infection. Expression of STAT5b correlated with advanced tumor stages and poor patient survival, and was more prominent in HBV-related HCC as compared to non-hepatitis B and non-hepatitis C type HCC [54]. Cell culture studies revealed that the HBV-encoded protein HBX caused the upregulation of STAT5b in HCC cell lines which was associated with the repression of E-cadherin, induction of EMT 
and increased cell invasion. Thus, STAT5b activation together with HBX enhances HCC aggressiveness by mechanisms involving EMT.

The resistance to chemotherapeutic treatment has been assessed in a large number of human HCC cell lines classified as either of epithelial or mesenchymal phenotype. In particular, epithelial cell lines were found to be more susceptible to EGFR-targeting therapeutics such as the small molecule tyrosine kinase inhibitor Erlotinib or Cetuximab, a monoclonal antibody against EGF-R, whereas mesenchymal cell lines showed resistance due to a high activity of PI3K/Akt and elevated levels of integrin-linked kinase (ILK). Remarkably, inhibition of ILK in mesenchymal cells resulted in reduced activation of Akt and partial MET which was sufficient for the increased sensitivity to EGF-R inhibitors. Thus, EMT is an important determinant for the sensitivity of HCC to EGFR-targeted therapies [55].

\section{Conclusions}

Currently, there is a considerable body of literature available indicating that hepatocellular EMT is a crucial event in HCC progression which causes an increase in malignancy of hepatocytes associating with tumor cell invasion and metastasis. Furthermore, evidence is accumulating that the mechanisms of EMT are used to generate myofibroblasts during chronic inflammation and hepatic fibrosis showing that the hepatocyte lineage contributes to the progression of fibrosis. Well-defined and advanced hepatocellular models of EMT have provided fundamental insights into the genetic programs underlying the changes in epithelial plasticity of neoplastic hepatocytes. Cell culture based strategies and gene-targeted mice therefore represent versatile and essential tools to mechanistically decipher individual signaling pathways and to identify inactivation of tumor suppressors and functional oncoprotein circuits involved in HCC cell invasion and metastasis. Insights into the molecular circuits of $\mathrm{HCC}$ in patients are of particular relevance for the validation of data obtained in cellular models, and vice versa, EMT-relevant data observed in HCC patients need to be further mechanistically analyzed in hepatocellular tumor models. Hence, translational approaches bridging rodent to human hepatocarcinogenesis are therefore inevitable to identify and characterize key regulatory components of hepatocellular EMT.

\section{Future perspective}

The transition of well differentiated carcinoma in situ to poorly differentiated invasive carcinoma is a predominant event in aggressive tumor progression of human HCC, leading to (i) local tissue invasion of malignant hepatocytes, (ii) intravasation into the vascular system, and (iii) extravasation and metastastic colonization. For HCC, intrahepatic metastasis is most frequent as the liver microenvironment shows a dense vasculature and is the competent milieu for HCC cells in the metastasis cascade. In this regard, EMT has been recognized as the initial phase of carcinoma cell evasion from well-organized structures, and as a prerequisite for the release of cells in order to spread into intrahepatic sites or even distal locations. The increasing availability of novel epithelial and mesenchymal markers must be recognized as valuable tools for the pathological assessment of human HCC samples. For instance, the dissociation of E-cadherin $/ \beta$-catenin complexes through downregulation or loss of E-cadherin might be a valuable biological marker for predicting tumor invasion and metastasis [42]. In this respect, it must be particularly emphasized that the EMT of HCC is under the control of the tumor microenvironment as reported by the cadherin switch, the nuclear accumulation of $\beta$-catenin at the tumor edge of HCC samples and in experimental models focusing on the interaction between the tumor and myofibroblasts of the stroma (Van Zijl et al., 2009, manuscript submitted; [45,48,56]). Further studies analyzing large cohorts of randomly selected HCC samples from different gradings and stagings have to be performed in order to evaluate the disruption of $\mathrm{E}$ - 
cadherin/ $\beta$-catenin complexes for clinical application. In the same line, additional studies should focus on the TGF- $\beta$-dependent phosphorylation of $\beta 1$ integrin, TM4SF-mediated cytosolic sequestration of $\mathrm{p} 27^{\mathrm{Kip} 1}$ or the expression of Sox 4 and miR-122/ADAM17 in order to assess their diagnostic and prognostic values for HCC progression [47,49-51]. Moreover, it is a matter of future studies whether novel inducers of EMT such as ILEI showing a particular cytoplasmic localization in neoplastic hepatocytes of poorly differentiated human HCC samples - can be used as a biomarker of HCC progression [39].

The list of molecular mechanisms causing EMT is still growing and currently appears as the tip of the iceberg. Future efforts using hypothesis-driven EMT models will allow further identification and detailed functional characterization of promising EMT regulatory molecules amenable to therapeutic intervention, and hence will facilitate the development of novel strategies to efficiently combat HCC progression. So far, the efficacy of the multikinase inhibitor Sorafenib - one available therapeutic option to efficiently interfere with HCC progression [57] - in EMT inhibition of HCC patients remains to be elucidated.

Another widely discussed research topic is the acquisition of stem cell traits during EMT which is suggested to contribute to the intratumoral heterogeneity, resulting in subpopulations of tumor cells showing differentiated features and other subpopulations displaying cancer stem cell (CSC)-like characteristics [18]. As described in other carcinoma types such as those derived from hair follicles in the skin, the mammary gland or colon, the adoption of cancer stemness associates with asynchronous cell division, resistance against therapeutic interventions and abilities for local cell invasion and metastasis. While a clear correlation between EMT and cancer stemness in HCC progression is currently missing, the involvement of EMT in intrahepatic metastasis and the insensitivity to targeted therapies point to such cellular mechanisms. Analysis of HCC patient samples showed that a high percentage of nuclear $\beta$-catenin accumulation in human HCC correlates with vascular invasion and recurrence of disease after liver transplantation (Zulehner et al., 2009, manuscript submitted). These data suggest that $\beta$-catenin signaling induces an immature malignant phenotype of hepatocyte progenitors that is responsible for the recurrence of human HCC after liver transplantation. Importantly, a relevant correlation between EMT and the adoption of cancer stemness has been demonstrated, since a subpopulation of $\mathrm{CD} 44^{\text {high }} / \mathrm{CD} 24^{\text {low }}$ cells could be identified as CSCs in breast tumorigenesis [58]. In the same line, colon carcinoma cells which exhibit nuclear $\beta$-catenin and locate at the invasive front for migrating into the stromal tissue, have been associated with EMT and cancer stemness [59]. In HCC, a substantial number (ranging from 28 to 50\%) of patients express markers of progenitor/biliary cells such as CK7, CK19 and OV6 suggesting a progenitor cell origin [60]. Indeed, expression of CK19 in HCC has been associated with a poor prognosis, aggressive clinical course and frequent recurrence after surgical treatment. Furthermore, $\mathrm{CD} 90^{+} / \mathrm{CD} 45^{-}$cells have been identified as CSCs in HCC and detected in liver cancer tissues and blood samples of HCC patients [61], whereas Ma et al. proposed CD133+ as a viable CSC marker since $\mathrm{CD} 133^{+}$cells exhibit both cancer and stemness features and contribute to chemoresistance through activation of PKB/Akt and Bcl-2 [62,63]. Yet, further studies are needed to analyze a conceivable correlation between the trans-differentiation of neoplastic hepatocytes to hepatic CSCs and EMT during HCC progression.

EMT has been critically and controversially discussed as the key process in carcinoma aggressiveness and metastasis [64]. In HCC, increasing evidence indicates a central role of EMT which might occur at the leading edge of tumor cells under the particular control of extrinsic factors derived from the tumor microenvironment in a paracrine fashion (Figure 4). So far, fundamental data are missing which demonstrate that the process of EMT is reversed to MET after intrahepatic or even distal metastatic colonization (Figure 4). The reversible and transient EMT-MET cycle would explain the limited opportunities to observe these 
changes in epithelial plasticity using histopathological analyses. Future studies will clarify this open issue and help to develop effective therapies against HCC.

\section{Executive summary}

\section{General aspects of EMT in embryonic development and adulthood}

- The epithelial to mesenchymal transition (EMT) represents a pivotal process during embryonic tissue remodeling and is essentially involved in gastrulation.

- EMT collectively associates with epithelial trans-differentiation, transient loss of cell-to-cell contacts and an enhanced motility during tissue repair and cancer in the adult.

\section{EMT during chronic liver inflammation and hepatic fibrosis}

- Hepatocytes induced to EMT by TGF- $\beta$ can be a potential source of fibroblasts in liver fibrosis. EMT can be blocked by BMP-7 or by antagonizing TGF- $\beta$ / Smad signaling.

- EMT results in the escape of apoptosis through activation of PI3K/Akt signaling.

\section{EMT tumor models of rodent hepatocytes}

- The synergy of TGF- $\beta$ and oncogenic Ras induces EMT of murine hepatocytes leading to autocrine regulation of TGF- $\beta$ signaling and activation of PDGF/ PDGF-R components.

- $\quad$ PDGF/PDGF-R signaling results in the nuclear accumulation of $\beta$-catenin which can induce a phenotype of hepatic progenitor cells.

\section{Molecular mechanisms of EMT in human HCC}

- Ln-5 plus TGF- $\beta$ cause EMT and nuclear accumulation of $\beta$-catenin. Induction of snail, slug and twist is implicated in the downregulation of E-cadherin.

- Phosphorylation of $\beta 1$ integrin by TGF- $\beta$ I receptor induces vascular invasion.

- The EMT inducer tetraspanin TM4SF, Sox 4 and its target genes neuropilin 1 and semaphorin $3 \mathrm{C}$ as well as the downregulation of $m i R-122$ and the corresponding increase of the sheddase ADAM17 are central events in the intrahepatic metastastasis of HCC.

- HCV core proteins and HBV-encoded HBX collaborate with TGF- $\beta / S m a d 3$ or STAT5b, respectively, to induce EMT and invasion of HCC.

\section{Pathophysiological role of EMT in human HCC progression}

- EMT of neoplastic hepatocytes is considered as a central event in intrahepatic dissemination and distal metastasis.

- EMT predicts sensitivity to EGFR-targeted therapies involving ILK.

- HCC progression might associate with cancer stemness and tumor reccurrence via mechanisms of EMT.

\section{Bibliography}

\section{Annotations of papers}


Papers of special note have been highlighted as either of interest $(\bullet)$ or of considerable interest $(\bullet \bullet)$ to readers.

\section{Zeisberg et al., 2007 (••)}

An important investigation demonstrating the conversion of hepatocytes to fibroblasts and the contribution of the hepatocyte lineage to the progression of fibrosis in vivo.

\section{Dooley et al., $2008(\bullet \bullet)$}

The paper highlights the essential role of TGF- $\beta$ signaling in the EMT of adult hepatocytes to fibroblastoid cells. Expression of Smad7 in hepatocytes of transgenic mice attenuated TGF- $\beta$ signaling and EMT.

\section{Murillo et al., 2005 (•)}

The study provides important insights into the molecular mechanisms of EMT resulting in the resistance against TGF- $\beta$-mediated apoptosis.

\section{Gotzmann et al., $2002(\bullet)$}

The authors show that the collaboration of TGF- $\beta$ with oncogenic Ras is a central event for the EMT of polarized but malignant murine hepatocytes. This hepatocellular tumor model mimics several important aspects of human HCC.

\section{Fransvea et al., $2009(\bullet)$}

The study provides clear evidence for the TGF- $\beta$ I receptor-mediated phosphorylation of $\beta 1$ integrin which stimulates the vascular invasion of HCC cells.

\section{Lee et al., $2008(\bullet \bullet)$}

A thorough study revealing for the first time the role of tetraspanin TM4SF5 as an inducer of EMT in human HCC. The authors show that TM4SF5 enhances cytosolic p27 Kip1 which inactivates RhoA and triggers EMT by loss of E-cadherin and gain of a-SMA.

\section{Battaglia et al., $2009(\bullet \bullet)$}

An elegant demonstration of the role of HCC-derived HCV core proteins on liver cancer progression through the cooperation with TGF- $\beta$ signaling. The study shows that the HCV core protein shifts the tumor-suppressive function of TGF- $\beta$ to EMT and HCC progression.

\section{Fuchs et al., $2008(\bullet)$}

The authors provide evidence that EMT predicts HCC sensitivity to EGFR-targeted therapies and show that ILK is a novel target to overcome HCC resistance to EGFR inhibition.

\section{Abbreviations}

$\begin{array}{ll}\text { BMP } & \text { bone morphogenic protein } \\ \text { CK } & \text { cytokeratin } \\ \text { CSC } & \text { cancer stem cells }\end{array}$




\begin{tabular}{ll} 
ECM & extracellular matrix \\
EMT & epithelial to mesenchymal transition \\
EGF & epidermal growth factor \\
FAK & focal adhesion kinase \\
FSP-1 & fibroblast-specific protein-1 \\
HBV & hepatitis B virus \\
HCV & hepatitis C virus \\
HCC & hepatocellular carcinoma \\
HNF & hepatocyte nuclear factor \\
HSC & hepatic stellate cell \\
ILEI & interleukin-like EMT inducer \\
ILK & integrin-linked kinase \\
Ln-5 & laminin-5 \\
MAPK & mitogen activated protein kinase \\
M2-PK & M2-pyruvate kinase \\
MET & mesenchymal to epithelial transition \\
MFB & myofibroblast \\
miR & microRNA \\
PI3K & phosphoinositide-3 kinase \\
PDGF & platelet-derived growth factor \\
STAT & signal transducer and activator of transcription \\
TGF & transforming growth factor \\
\hline
\end{tabular}

\section{Bibliography}

1. El-Serag HB, Rudolph KL. Hepatocellular carcinoma: epidemiology and molecular carcinogenesis. Gastroenterology. 2007; 132:2557-2776. [PubMed: 17570226]

2. Kensler TW, Qian GS, Chen JG, Groopman JD. Translational strategies for cancer prevention in liver. Nat Rev Cancer. 2003; 3:321-329. [PubMed: 12724730]

3. Farazi PA, DePinho RA. Hepatocellular carcinoma pathogenesis: from genes to environment. Nat Rev Cancer. 2006; 6:674-687. [PubMed: 16929323]

4. Friedman SL. Mechanisms of hepatic fibrogenesis. Gastroenterology. 2008; 134:1655-1669. [PubMed: 18471545]

5. Jou J, Choi SS, Diehl AM. Mechanisms of disease progression in nonalcoholic fatty liver disease. Semin Liver Dis. 2008; 28:370-379. [PubMed: 18956293]

6. Wallace DF, Subramaniam VN. Co-factors in liver disease: The role of HFE-related hereditary hemochromatosis and iron. Biochim Biophys Acta. 2008

7. Teufel A, Staib F, Kanzler S, Weinmann A, Schulze-Bergkamen H, Galle PR. Genetics of hepatocellular carcinoma. World J Gastroenterol. 2007; 13:2271-2282. [PubMed: 17511024]

8. Villanueva A, Newell P, Chiang DY, Friedman SL, Llovet JM. Genomics and signaling pathways in hepatocellular carcinoma. Semin Liver Dis. 2007; 27:55-76. [PubMed: 17295177]

9. Sun VC, Sarna L. Symptom management in hepatocellular carcinoma. Clin J Oncol Nurs. 2008; 12:759-766. [PubMed: 18842532] 
10. Llovet JM, Bruix J. Molecular targeted therapies in hepatocellular carcinoma. Hepatology. 2008; 48:1312-1327. [PubMed: 18821591]

11. Michalopoulos GK, DeFrances MC. Liver regeneration. Science. 1997; 276:60-66. [PubMed: 9082986]

12. Taub R. Liver regeneration: from myth to mechanism. Nat Rev Mol Cell Biol. 2004; 5:836-847. [PubMed: 15459664]

13. Overturf K, al-Dhalimy M, Ou CN, Finegold M, Grompe M. Serial transplantation reveals the stem-cell-like regenerative potential of adult mouse hepatocytes. Am J Pathol. 1997; 151:12731280. [PubMed: 9358753]

14. Breuhahn K, Longerich T, Schirmacher P. Dysregulation of growth factor signaling in human hepatocellular carcinoma. Oncogene. 2006; 25:3787-3800. [PubMed: 16799620]

15. Tannapfel A, Busse C, Weinans L, et al. INK4a-ARF alterations and p53 mutations in hepatocellular carcinomas. Oncogene. 2001; 20:7104-7109. [PubMed: 11704835]

16. Friedl P, Wolf K. Tumour-cell invasion and migration: diversity and escape mechanisms. Nat Rev Cancer. 2003; 3:362-374. [PubMed: 12724734]

17. Gotzmann J, Mikula M, Eger A, et al. Molecular aspects of epithelial cell plasticity: implications for local tumor invasion and metastasis. Mutat Res. 2004; 566:9-20. [PubMed: 14706509]

18. Polyak K, Weinberg RA. Transitions between epithelial and mesenchymal states: acquisition of malignant and stem cell traits. Nat Rev Cancer. 2009; 9:265-273. [PubMed: 19262571]

19. Thiery JP, Sleeman JP. Complex networks orchestrate epithelial-mesenchymal transitions. Nat Rev Mol Cell Biol. 2006; 7:131-142. [PubMed: 16493418]

20. Grunert S, Jechlinger M, Beug H. Diverse cellular and molecular mechanisms contribute to epithelial plasticity and metastasis. Nat Rev Mol Cell Biol. 2003; 4:657-665. [PubMed: $12923528]$

21. Thiery JP. Epithelial-mesenchymal transitions in tumour progression. Nat Rev Cancer. 2002; 2:442-454. [PubMed: 12189386]

22. Bissell MJ, Radisky D. Putting tumours in context. Nature Rev Cancer. 2001; 1:46-54. [PubMed: 11900251]

23. Iredale JP. Models of liver fibrosis: exploring the dynamic nature of inflammation and repair in a solid organ. J Clin Invest. 2007; 117:539-548. [PubMed: 17332881]

24. Zeisberg M, Yang C, Martino M, et al. Fibroblasts derive from hepatocytes in liver fibrosis via epithelial to mesenchymal transition. J Biol Chem. 2007; 282:23337-23347. [PubMed: 17562716]

25. Dooley S, Hamzavi J, Ciuclan L, et al. Hepatocyte-specific Smad7 expression attenuates TGFbeta-mediated fibrogenesis and protects against liver damage. Gastroenterology. 2008; 135:642 659. [PubMed: 18602923]

26. Kaimori A, Potter J, Kaimori JY, Wang C, Mezey E, Koteish A. Transforming growth factor-beta1 induces an epithelial-to-mesenchymal transition state in mouse hepatocytes in vitro. J Biol Chem. 2007; 282:22089-22101. [PubMed: 17513865]

27. Godoy P, Hengstler JG, Ilkavets I, et al. Extracellular matrix modulates sensitivity of hepatocytes to fibroblastoid dedifferentiation and transforming growth factor beta-induced apoptosis. Hepatology. 2009; 49:1-13. [PubMed: 19108004]

28. Cicchini C, Laudadio I, Citarella F, et al. TGFbeta-induced EMT requires focal adhesion kinase (FAK) signaling. Exp Cell Res. 2008; 314:143-152. [PubMed: 17949712]

29. Nitta T, Kim JS, Mohuczy D, Behrns KE. Murine cirrhosis induces hepatocyte epithelial mesenchymal transition and alterations in survival signaling pathways. Hepatology. 2008; 48:909919. [PubMed: 18712785]

30. Murillo MM, del Castillo G, Sanchez A, Fernandez M, Fabregat I. Involvement of EGF receptor and c-Src in the survival signals induced by TGF-beta1 in hepatocytes. Oncogene. 2005; 24:45804587. [PubMed: 15856020]

31. Valdes F, Alvarez AM, Locascio A, et al. The epithelial mesenchymal transition confers resistance to the apoptotic effects of transforming growth factor Beta in fetal rat hepatocytes. Mol Cancer Res. 2002; 1:68-78. [PubMed: 12496370] 
32. Lazarevich NL, Cheremnova OA, Varga EV, et al. Progression of HCC in mice is associated with a downregulation in the expression of hepatocyte nuclear factors. Hepatology. 2004; 39:10381047. [PubMed: 15057908]

33. Amicone L, Spagnoli FM, Spath G, et al. Transgenic expression in the liver of truncated Met blocks apoptosis and permits immortalization of hepatocytes. EMBO J. 1997; 16:495-503. [PubMed: 9034332]

34. Mikula M, Fuchs E, Huber H, Beug H, Schulte-Hermann R, Mikulits W. Immortalized p19ARF null hepatocytes restore liver injury and generate hepatic progenitors after transplantation. Hepatology. 2004; 39:628-634. [PubMed: 14999681]

35. Gotzmann J, Fischer AN, Zojer M, et al. A crucial function of PDGF in TGF-beta-mediated cancer progression of hepatocytes. Oncogene. 2006; 25:3170-3185. [PubMed: 16607286]

36. Gotzmann J, Huber H, Thallinger C, et al. Hepatocytes convert to a fibroblastoid phenotype through the cooperation of TGF-beta1 and Ha-Ras: steps towards invasiveness. J Cell Sci. 2002; 115:1189-1202. [PubMed: 11884518]

37. Fischer AN, Herrera B, Mikula M, et al. Integration of Ras subeffector signaling in TGF- $\{$ beta $\}$ mediated late stage hepatocarcinogenesis. Carcinogenesis. 2005:931-942. [PubMed: 15705598]

38. Mikula M, Gotzmann J, Fischer AN, et al. The proto-oncoprotein c-Fos negatively regulates hepatocellular tumorigenesis. Oncogene. 2003; 22:6725-6738. [PubMed: 14555986]

39. Lahsnig C, Mikula M, Petz M, et al. ILEI requires oncogenic Ras for the epithelial to mesenchymal transition of hepatocytes and liver carcinoma progression. Oncogene. 2009; 28:638-650. [PubMed: 19015638]

40. Fischer AN, Fuchs E, Mikula M, Huber H, Beug H, Mikulits W. PDGF essentially links TGF-beta signaling to nuclear beta-catenin accumulation in hepatocellular carcinoma progression. Oncogene. 2007; 26:3395-3405. [PubMed: 17130832]

41. Lee HC, Kim M, Wands JR. Wnt/Frizzled signaling in hepatocellular carcinoma. Front Biosci. 2006; 11:1901-1915. [PubMed: 16368566]

42. Zhai B, Yan HX, Liu SQ, Chen L, Wu MC, Wang HY. Reduced expression of E-cadherin/catenin complex in hepatocellular carcinomas. World J Gastroenterol. 2008; 14:5665-5673. [PubMed: 18837082]

43. Fransvea E, Angelotti U, Antonaci S, Giannelli G. Blocking transforming growth factor-beta upregulates E-cadherin and reduces migration and invasion of hepatocellular carcinoma cells. Hepatology. 2008; 47:1557-1566. [PubMed: 18318443]

44. Lee TK, Poon RT, Yuen AP, et al. Twist overexpression correlates with hepatocellular carcinoma metastasis through induction of epithelial-mesenchymal transition. Clin Cancer Res. 2006; 12:5369-5376. [PubMed: 17000670]

45. Giannelli G, Bergamini C, Fransvea E, Sgarra C, Antonaci S. Laminin-5 with transforming growth factor-beta1 induces epithelial to mesenchymal transition in hepatocellular carcinoma. Gastroenterology. 2005; 129:1375-1383. [PubMed: 16285938]

46. Bergamini C, Sgarra C, Trerotoli P, et al. Laminin-5 stimulates hepatocellular carcinoma growth through a different function of alpha6beta4 and alpha3beta1 integrins. Hepatology. 2007; 46:1801-1809. [PubMed: 17948258]

47. Fransvea E, Mazzocca A, Antonaci S, Giannelli G. Targeting transforming growth factor (TGF)betaRI inhibits activation of beta1 integrin and blocks vascular invasion in hepatocellular carcinoma. Hepatology. 2009; 49:839-850. [PubMed: 19115199]

48. Riou P, Saffroy R, Chenailler C, et al. Expression of T-cadherin in tumor cells influences invasive potential of human hepatocellular carcinoma. FASEB J. 2006; 20:2291-2301. [PubMed: 17077306]

49. Lee SA, Lee SY, Cho IH, et al. Tetraspanin TM4SF5 mediates loss of contact inhibition through epithelial-mesenchymal transition in human hepatocarcinoma. J Clin Invest. 2008; 118:13541366. [PubMed: 18357344]

50. Liao YL, Sun YM, Chau GY, et al. Identification of SOX4 target genes using phylogenetic footprinting-based prediction from expression microarrays suggests that overexpression of SOX4 potentiates metastasis in hepatocellular carcinoma. Oncogene. 2008:5578-5589. [PubMed: 18504433] 
51. Tsai WC, Hsu PW, Lai TC, et al. MicroRNA-122, a tumor suppressor microRNA that regulates intrahepatic metastasis of hepatocellular carcinoma. Hepatology. 2009; 49:1571-1582. [PubMed: 19296470]

52. Yuki K, Hirohashi S, Sakamoto M, Kanai T, Shimosato Y. Growth and spread of hepatocellular carcinoma. A review of 240 consecutive autopsy cases. Cancer. 1990; 66:2174-2179. [PubMed: 2171748]

53. Battaglia S, Benzoubir N, Nobilet $S$, et al. Liver cancer-derived hepatitis $C$ virus core proteins shift TGF-Beta responses from tumor suppression to epithelial-mesenchymal transition. PLoS ONE. 2009; 4:e4355. [PubMed: 19190755]

54. Lee TK, Man K, Poon RT, et al. Signal transducers and activators of transcription 5b activation enhances hepatocellular carcinoma aggressiveness through induction of epithelial-mesenchymal transition. Cancer Res. 2006; 66:9948-9956. [PubMed: 17047057]

55. Fuchs BC, Fujii T, Dorfman JD, et al. Epithelial-to-mesenchymal transition and integrin-linked kinase mediate sensitivity to epidermal growth factor receptor inhibition in human hepatoma cells. Cancer Res. 2008; 68:2391-2399. [PubMed: 18381447]

56. Mikula M, Proell V, Fischer AN, Mikulits W. Activated hepatic stellate cells induce tumor progression of neoplastic hepatocytes in a TGF-beta dependent fashion. J Cell Physiol. 2006; 209:560-567. [PubMed: 16883581]

57. Llovet JM, Ricci S, Mazzaferro V, et al. Sorafenib in advanced hepatocellular carcinoma. N Engl J Med. 2008; 359:378-390. [PubMed: 18650514]

58. Mani SA, Guo W, Liao MJ, et al. The epithelial-mesenchymal transition generates cells with properties of stem cells. Cell. 2008; 133:704-715. [PubMed: 18485877]

59. Fodde R, Brabletz T. Wnt/beta-catenin signaling in cancer stemness and malignant behavior. Curr Opin Cell Biol. 2007; 19:150-158. [PubMed: 17306971]

60. Roskams T. Liver stem cells and their implication in hepatocellular and cholangiocarcinoma. Oncogene. 2006; 25:3818-3822. [PubMed: 16799623]

61. Yang ZF, Ngai P, Ho DW, et al. Identification of local and circulating cancer stem cells in human liver cancer. Hepatology. 2008; 47:919-928. [PubMed: 18275073]

62. Ma S, Chan KW, Hu L, et al. Identification and characterization of tumorigenic liver cancer stem/ progenitor cells. Gastroenterology. 2007; 132:2542-2556. [PubMed: 17570225]

63. Ma S, Lee TK, Zheng BJ, Chan KW, Guan XY. CD133+ HCC cancer stem cells confer chemoresistance by preferential expression of the Akt/PKB survival pathway. Oncogene. 2008; 27:1749-1758. [PubMed: 17891174]

64. Thompson EW, Newgreen DF, Tarin D. Carcinoma invasion and metastasis: a role for epithelialmesenchymal transition? Cancer Res. 2005; 65:5991-5995. discussion 5995. [PubMed: 16024595] 


\section{Embryonic development \\ Organogenesis}

Wound healing

Fibrosis

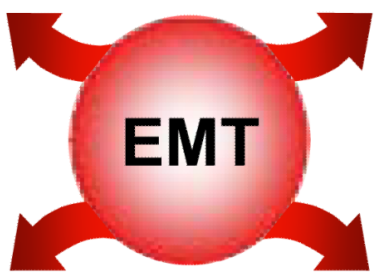

\section{Stemness \\ Cancer stem cells}

\section{Invasion \\ Tumor progression}

Figure 1.

The involvement of EMT in physiological and pathophysiological events. 

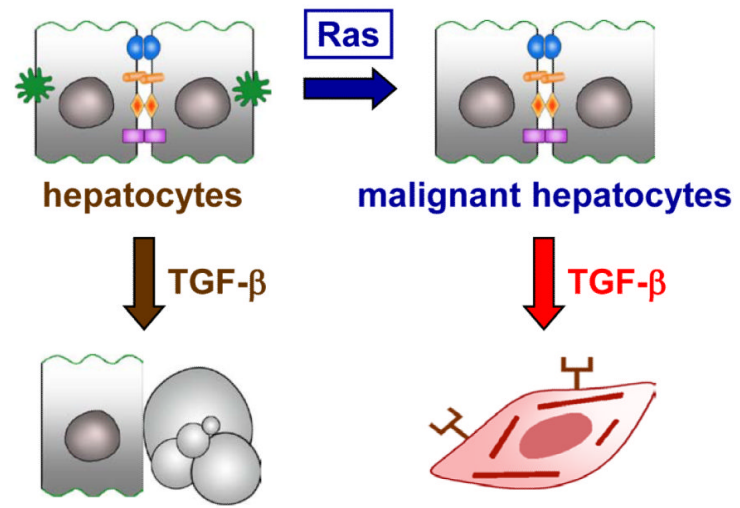

cell cycle arrest

$\&$ apoptosis

EMT \& tumor

progression



Figure 2. TGF- $\beta$ causes EMT of malignant hepatocytes in cooperation with oncogenic Ras activation

TGF- $\beta$, transforming growth factor- $\beta$. 


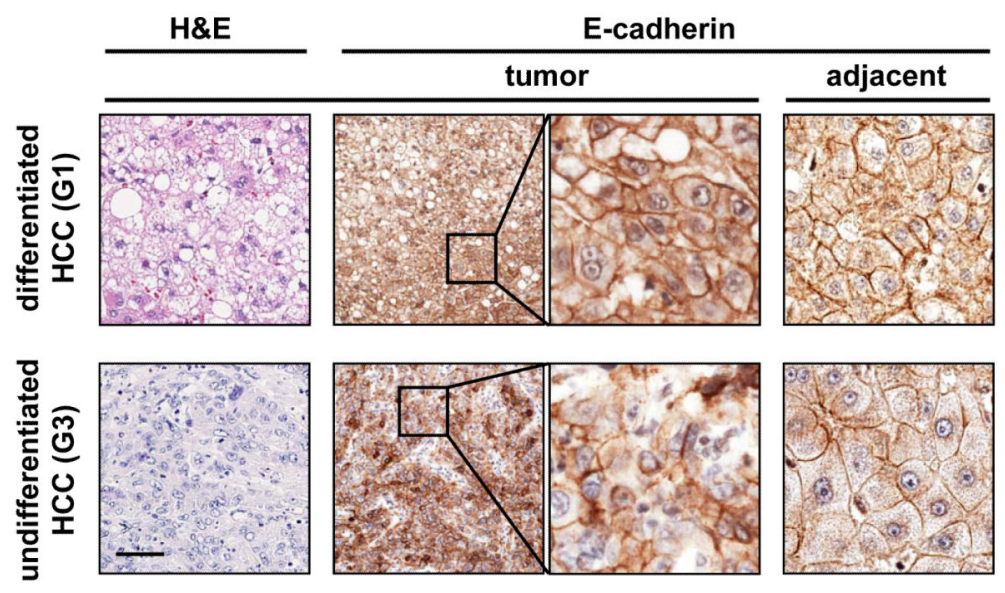

Figure 3. Poorly differentiated human HCC show loss of epithelial markers such as plasma membrane-bound E-cadherin, suggesting EMT

Human HCC samples were immunohistochemically stained with anti-E-cadherin (Ecadherin) antibody. Differentiated HCC (histological grading G1) show E-cadherin at cell boundaries, whereas poorly differentiated human HCC (histological grading G3) show loss or cytoplasmic dislocation of E-cadherin expression at plasma membranes. Black boxes indicate regions at 4-fold magnification. Two representative HCC samples are shown. Bar, $50 \mu \mathrm{m}$. 


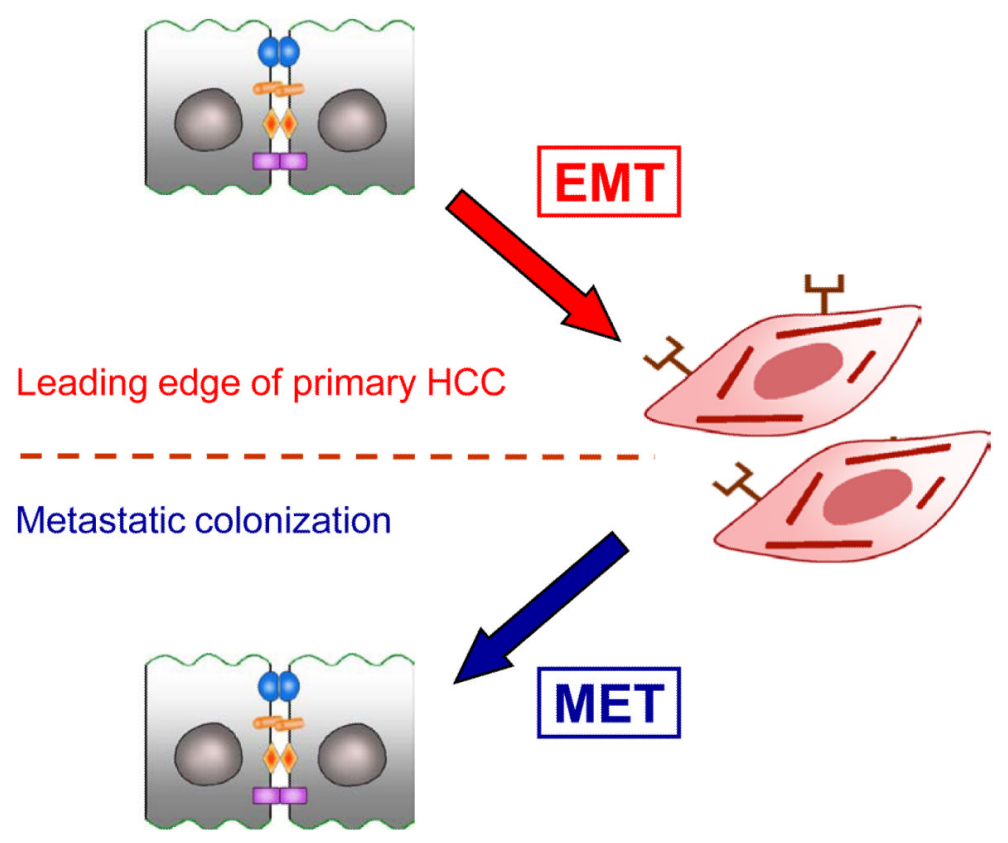

Figure 4. The putative EMT-MET cycle suggests transient and reversible changes in the epithelial plasticity during tumor progression MET, mesenchymal to epithelial transition. 